Physics

Physics Research Publications

\title{
Effect of temperature on isotopic mass dependence of excitonic band gaps in semiconductors: $\mathrm{ZnO}$
}
H. Alawadhi*
S. Tsoi ${ }^{\dagger} \quad$ X. $\mathrm{Lu}^{\ddagger}$
A. K. Ramdas**
M. Grimsditch ${ }^{\dagger \dagger}$
M. Cardona
R. Lauck $^{\S}$

$*$
$\dagger$
$\ddagger$
$* *$
$\dagger \dagger$
$\ddagger \ddagger$
$\S$

This paper is posted at Purdue e-Pubs.

http://docs.lib.purdue.edu/physics_articles/737 


\title{
Effect of temperature on isotopic mass dependence of excitonic band gaps in semiconductors: $\mathrm{ZnO}$
}

\author{
H. Alawadhi \\ Department of Basic Sciences, University of Sharjah, United Arab Emirates \\ S. Tsoi, ${ }^{*}$ X. Lu, and A. K. Ramdas \\ Department of Physics, Purdue University, West Lafayette, Indiana 47907, USA \\ M. Grimsditch \\ Argonne National Laboratory, Argonne, Illinois 60439, USA \\ M. Cardona and R. Lauck \\ Max-Planck-Institut für Festkörperforschung, Heisenbergstrasse 1, D-70569 Stuttgart, Germany
}

(Received 24 December 2006; published 29 May 2007)

\begin{abstract}
The temperature dependence of the $A, B$, and $C$ excitons of $\mathrm{ZnO}$, observed in modulated reflectivity spectra of ${ }^{68} \mathrm{Zn}^{18} \mathrm{O}$ and ${ }^{n a t} \mathrm{Zn}^{\text {nat }} \mathrm{O}$ in the range $10-400 \mathrm{~K}$, reveal the superposition of band-gap renormalization originating in electron-phonon interaction and volume changes associated with thermal expansion and (or) isotopic composition in combination with anharmonicity. At low temperatures, the $A, B$, and $C$ excitons in natural $\mathrm{ZnO}$ reach limiting values depressed from their values for the infinitely massive isotopes (the latter are free from electron-phonon interaction and anharmonicity). The $\mathrm{C}$ excitons of ${ }^{68} \mathrm{Zn}^{18} \mathrm{O}$ and ${ }^{\text {nat }} \mathrm{Zn}^{\text {nat }} \mathrm{O}$ converge with increasing temperature, demonstrating the independence of the band gap from isotopic mass at high temperatures.
\end{abstract}

DOI: 10.1103/PhysRevB.75.205207

PACS number(s): 78.55.Et, 63.20.Kr, 78.40.Fy

\section{INTRODUCTION}

The temperature dependence of band gaps of the tetrahedrally coordinated semiconductors has been the subject of many experimental and theoretical studies ever since the original discovery of the absorption edge of $\mathrm{Si}$ and its shift with temperature by Becker and Fan. ${ }^{1}$ It has been theoretically identified ${ }^{2-4}$ to arise from the superposition of two effects: thermal expansion of the crystal and electron-phonon interaction (at constant volume), both effects being dependent on the thermal population of the phonon modes. In this context, the measurement of a band gap $\left(E_{G}\right)$ shift with temperature provides an ideal test of theories of electron-phonon interaction. ${ }^{5}$ In addition, the temperature dependence of the band gap can yield $E_{G}^{\infty}$, its hypothetical value in the absence of phonons, i.e., for a static lattice composed of the "infinitely" massive isotopes of the constituent atoms. This information is invaluable for testing ab initio calculations of the electronic band structure. ${ }^{6}$

Closely related to the temperature dependence of the band gap is its variation with isotopic masses of atoms composing the semiconductor. At a fixed temperature, the population of phonon modes is governed by the isotopic masses, resulting in an isotopic dependence of the band gap via volume expansion as well as electron-phonon interaction. With the recent access to isotopically engineered semiconductors, lowtemperature spectroscopic measurements of band gaps of several elemental ${ }^{7-12}$ and binary ${ }^{13}$ tetrahedrally coordinated semiconductors have been the object of study. They have yielded with great precision the zero-point renormalization of the band gaps, i.e., the deviation of the band gap at absolute zero from that in the static lattice caused by the quantum vibrational amplitudes at $T=0$.
Theoretical considerations ${ }^{5,9}$ have predicted an interesting interplay between the temperature and the isotopic mass dependence: the latter is most pronounced at low temperatures and tends to zero above the Debye temperature. The present work addresses this prediction experimentally.

$\mathrm{ZnO}$ is a wide band-gap II-VI wurtzite semiconductor, attractive for a variety of technological applications. ${ }^{14}$ Previously, the temperature dependence of its $A, B$, and $C$ excitonic band gaps, associated with the lowest conduction band and the top three valence bands at the center of the Brillouin zone, has been studied from 4.2 to $300 \mathrm{~K}$ employing reflectivity, ${ }^{15}$ photoluminescence (PL), ${ }^{15-17}$ and photoluminescence excitation spectroscopy (PLE). ${ }^{18}$ In addition, the isotopic dependence of the $\mathrm{ZnO}$ band gaps has been investigated at low temperature by wavelength-modulated reflectivity ${ }^{19,20}$ and PL. ${ }^{17}$ In this study we present results on the $A, B$, and $C$ excitonic band gaps of $\mathrm{ZnO}$ as a function of temperature. The sharp signatures observed by using photomodulation allowed us to extend the temperature range of experimental data to $400 \mathrm{~K}$ and deduce their zero-point renormalizations in ${ }^{n a t} \mathrm{Zn}^{\text {nat }} \mathrm{O}(\mathrm{ZnO}$ with natural isotopic composition of $\mathrm{Zn}$ and $\mathrm{O}$, i.e., ${ }^{65.4} \mathrm{Zn}^{16} \mathrm{O}$ ). In addition, the temperature dependence obtained for the $C$ excitonic band gap in an isotopically engineered ${ }^{68} \mathrm{Zn}^{18} \mathrm{O}$, together with similar data in ${ }^{n a t} \mathrm{Zn}^{\text {nat }} \mathrm{O}$, provide convincing experimental evidence for a vanishing isotopic dependence of the band gap at high temperatures. However, the effect of the isotopic mass on the thermal expansion has been shown to tend to zero at high $T$ in the case of germanium..$^{5,21}$

\section{EXPERIMENT}

Details about the samples $\left({ }^{n a t} \mathrm{Zn}^{n a t} \mathrm{O}\right.$ and $\left.{ }^{68} \mathrm{Zn}^{18} \mathrm{O}\right)$ and the growth procedure are given in Ref. 20. For the temperature 
dependence of excitons of the natural $\mathrm{ZnO}$, measurements were performed with unpolarized light using flat surfaces parallel to the $c$ axis. In the temperature range $4.2-295 \mathrm{~K}$, the sample was placed inside a continuous flow He cryostat. The experiments at temperatures higher than $295 \mathrm{~K}$ (room temperature) were performed with the sample inside an electrically heated oven, equipped with a fused quartz optical window. In both cases the temperature was regulated by a temperature controller.

In wavelength modulation, a sharp spectral feature, associated with the source, overlapped with the $A$ and $B$ excitonic features of $\mathrm{ZnO}$ above $150 \mathrm{~K}$. This limited the use of wavelength modulation to lower temperatures, at which excitonic features are spectrally shifted from those of the light source. Thus the contactless photomodulation was preferred for the high-temperature study.

\section{THEORETICAL BACKGROUND}

The dependence of the band gap $\left(E_{G}\right)$ on temperature for constant isotopic mass $(M)$ and pressure $(P)$ can be written as

$$
\left(\frac{\partial E_{G}}{\partial T}\right)_{M, P}=\left(\frac{\partial E_{G}}{\partial T}\right)_{M, V}+\frac{D}{V}\left(\frac{\partial V}{\partial T}\right)_{M, P} .
$$

Here $D=-B\left(\partial E_{G} / \partial P\right)_{T, M}$ is the deformation potential for hydrostatic strain and $B$ is the bulk modulus. The first term on the right-hand side of Eq. (1) results from the electronphonon interaction and the second is due to the thermal expansion of the crystal.

It follows from Eq. (1) that the band gap at temperature $T$ with respect to that at absolute zero is given by

$$
\begin{aligned}
E_{G}(T, M, P)-E_{G}(T=0, M, P) \\
\quad=\int_{0}^{T}\left(\frac{\partial E_{G}}{\partial T^{\prime}}\right)_{M, P} d T^{\prime}=\int_{0}^{T}\left(\frac{\partial E_{G}}{\partial T^{\prime}}\right)_{M, V} d T^{\prime} \\
\quad+\int_{0}^{T} \frac{D}{V}\left(\frac{\partial V}{\partial T^{\prime}}\right)_{M, P} d T^{\prime} .
\end{aligned}
$$

It is convenient to express $E_{G}(T=0, M, P)$ in terms of the band gap expected for the static lattice $E_{G}^{\infty}$ and its corresponding renormalization by zero-point vibrations,

$$
E_{G}(T=0, M, P)=E_{G}^{\infty}-\Delta E_{G}^{e-p h}-\Delta E_{G}^{v o l} .
$$

The superscript " $\infty$ " in $E_{G}^{\infty}$ denotes an infinite isotopic mass, which corresponds to the static lattice at any temperature. In Eq. (3), the zero-point renormalization is expressed as a sum of two terms, $\Delta E_{G}^{e-p h}$ and $\Delta E_{G}^{v o l}$, due to the electron-phonon interaction and the change in the volume caused by zeropoint vibrations, respectively.

Combining Eqs. (2) and (3), the temperature dependence of the band gap is given by

$$
\begin{aligned}
E_{G}(T, M, P)= & E_{G}^{\infty}-\left[\Delta E_{G}^{e-p h}-\int_{0}^{T}\left(\frac{\partial E_{G}}{\partial T^{\prime}}\right)_{M, V} d T^{\prime}\right] \\
& -\left[\Delta E_{G}^{v o l}-\int_{0}^{T} \frac{D}{V}\left(\frac{\partial V}{\partial T^{\prime}}\right)_{M, P} d T^{\prime}\right],
\end{aligned}
$$

where the term in the first square bracket is the band-gap renormalization due to electron-phonon interaction and that in the second square bracket represents the effect of the change in volume with temperature. An expression for the former can be found in Eq. (3) of Ref. 22, viz.

$$
\left[\Delta E_{G}^{e-p h}-\int_{0}^{T}\left(\frac{\partial E_{G}}{\partial T^{\prime}}\right)_{M, V} d T^{\prime}\right]=\sum_{j, \mathbf{q}}\left(\frac{\partial E_{G}}{\partial n_{j, \mathbf{q}}}\right)\left(n_{j, \mathbf{q}}+\frac{1}{2}\right),
$$

where $j$ and $\mathbf{q}$ denote the branch and wave vector of a phonon mode, respectively, its population $n_{j, \mathbf{q}}$ at temperature $T$ being determined by Bose-Einstein statistics. The summation in Eq. (5) is performed over all phonon modes.

In order to evaluate the renormalization due to the change in volume, we note that $\Delta E_{G}^{v o l}$ can be written as ${ }^{12}$

$$
\Delta E_{G}^{v o l}=\int_{M}^{\infty} \frac{D}{V}\left(\frac{\partial V}{\partial M^{\prime}}\right)_{T=0, P} d M^{\prime}=-D \ln \frac{V(T=0, M, P)}{V_{0}},
$$

where $V_{0}$ is the volume of the static lattice. Thus we obtain

$$
\left[\Delta E_{G}^{v o l}-\int_{0}^{T} \frac{D}{V}\left(\frac{\partial V}{\partial T^{\prime}}\right)_{M, P} d T^{\prime}\right]=-D \ln \frac{V(T, M, P)}{V_{0}} .
$$

An analytical expression for $V(T, M, P)$ is given in Eq. (2.3) of Ref. 23 as

$$
V(T, M, P)=V_{0}+\frac{1}{B} \sum_{j, \mathbf{q}} \hbar \Omega_{j}(\mathbf{q}) \gamma_{j}(\mathbf{q})\left(n_{j, \mathbf{q}}+\frac{1}{2}\right),
$$

where $\Omega_{j}(\mathbf{q})$ is the frequency of the phonon mode $j, \mathbf{q}$ and

$$
\gamma_{j}(\mathbf{q})=-\left.\frac{V_{0}}{\Omega_{j}(\mathbf{q})} \frac{\partial \Omega_{j}(\mathbf{q})}{\partial V}\right|_{V_{0}}
$$

is its mode Grüneisen parameter. In the temperature range employed in the present investigation, the second term on the right-hand side of Eq. (7) is much smaller than the first term and Eq. (6) can be approximated by

$$
\begin{aligned}
& {\left[\Delta E_{G}^{v o l}-\int_{0}^{T} \frac{D}{V}\left(\frac{\partial V}{\partial T^{\prime}}\right)_{M, P} d T^{\prime}\right]} \\
& \quad \approx-\frac{D}{B V_{0}} \sum_{j, \mathbf{q}} \hbar \Omega_{j}(\mathbf{q}) \gamma_{j}(\mathbf{q})\left(n_{j, \mathbf{q}}+\frac{1}{2}\right) .
\end{aligned}
$$

Combining Eqs. (4), (5), and (8), the temperature dependence of the band gap becomes 


$$
\begin{aligned}
E_{G}(T, M, P)= & E_{G}^{\infty}-\sum_{j, \mathbf{q}}\left(\frac{\partial E_{G}}{\partial n_{j, \mathbf{q}}}\right)\left(n_{j, \mathbf{q}}+\frac{1}{2}\right) \\
& +\frac{D}{B V_{0}} \sum_{j, \mathbf{q}} \hbar \Omega_{j}(\mathbf{q}) \gamma_{j}(\mathbf{q})\left(n_{j, \mathbf{q}}+\frac{1}{2}\right) .
\end{aligned}
$$

Calculation of the coefficients $\left(\partial E_{G} / \partial n_{j, \mathbf{q}}\right)$ and the Grüneisen parameters for all the phonon modes of a crystal is a formidable task requiring a good knowledge of the electron band structure and the lattice dynamics. A more popular and significantly simpler theoretical approach to the temperature dependence of the band gap involves a two-oscillator approximation. ${ }^{22,24,25}$ The approximation assumes that the lattice dynamics of the semiconductor can be described in a satisfactory manner by two harmonic oscillators, for instance, an average acoustic and an average optic mode.

In the case of $\mathrm{ZnO}$, with its large difference in the masses of the anion and the cation, the amplitude of the acoustic mode is dominated by the heavy mass $(\mathrm{Zn})$ and that of the optic mode by the light mass $(\mathrm{O})$, transforming Eq. (9) into

$$
\begin{aligned}
E_{G}(T, M, P)= & E_{G}^{\infty}-\left(\frac{p_{\mathrm{Zn}} A_{\mathrm{Zn}}}{\Omega_{\mathrm{Zn}} M_{\mathrm{Zn}}}-\frac{p_{\mathrm{Zn}} D \hbar \Omega_{\mathrm{Zn}} \gamma_{\mathrm{Zn}}}{B V_{0}}\right) \\
& \times\left(\frac{1}{\exp \left(\hbar \Omega_{\mathrm{Zn}} / k_{B} T\right)-1}+\frac{1}{2}\right) \\
& -\left(\frac{p_{\mathrm{O}} A_{\mathrm{O}}}{\Omega_{\mathrm{O}} M_{\mathrm{O}}}-\frac{p_{\mathrm{O}} D \hbar \Omega_{\mathrm{O}} \gamma_{\mathrm{O}}}{B V_{0}}\right) \\
& \times\left(\frac{1}{\exp \left(\hbar \Omega_{\mathrm{O}} / k_{B} T\right)-1}+\frac{1}{2}\right) .
\end{aligned}
$$

Here $\Omega_{\mathrm{Zn}}$ and $\Omega_{\mathrm{O}}$ are the average frequencies of the $\mathrm{Zn}$ dominated modes ${ }^{26}$ and the optic, O-dominated modes, respectively; $\gamma_{Z n}, \gamma_{O}$ are the corresponding average Grüneisen parameters; $p_{\mathrm{Zn}}, p_{\mathrm{O}}$ are the degeneracies of the zinclike and oxygenlike oscillators; $M_{\mathrm{Zn}}$ and $M_{\mathrm{O}}$ represent average isotopic masses of $\mathrm{Zn}$ and $\mathrm{O}$; and $A_{\mathrm{Zn}}$ and $A_{\mathrm{O}}$ are parameters ${ }^{22}$ describing the corresponding electron-phonon interactions. Conversion of Eq. (9) into Eq. (10) reduces $V_{0}$ to the volume of the primitive cell. This volume is twice as large in wurtzite as in zinc-blende, hence $p_{\mathrm{Zn}}=p_{\mathrm{O}}=6$ in wurtzite and $p_{\mathrm{Zn}}=p_{\mathrm{O}}=3$ in zinc-blende. In Eq. (10) the relationship ${ }^{22}$

$$
\frac{\partial E_{G}}{\partial n_{i}}=\frac{A_{i}}{\Omega_{i} M_{i}},
$$

where $i=\mathrm{Zn}, \mathrm{O}$ was used. This expression results from the renormalization of electronic energies by electron-phonon interaction treated as time-dependent perturbation with respect to the electronic levels of the static lattice. ${ }^{5}$ The interaction term linear in phonon displacement treated as a first-order perturbation yields zero renormalization. The largest contribution then arises from the linear term treated to the second order $\left(\right.$ Fan terms ${ }^{3}$ ) and a term quadratic in displacements treated to the first order (Debye-Waller terms ${ }^{4}$ ), both yielding renormalization quadratic in phonon amplitude, i.e., proportional to $\left(n_{i}+1 / 2\right) /\left(\Omega_{i} M_{i}\right){ }^{27}$ Thus the dependence of the band gap $E_{G}$ on the phonon population factor $n_{i}$ is given through Eq. (11) with the appropriate parameter $A_{i}$.
According to Eq. (10), at absolute zero the dependence of the band gap on isotopic mass is

$$
\begin{aligned}
E_{G}(T=0, M, P)= & E_{G}^{\infty}-\frac{1}{2}\left(\frac{p_{\mathrm{Zn}} A_{\mathrm{Zn}}}{\Omega_{\mathrm{Zn}} M_{\mathrm{Zn}}}-\frac{p_{\mathrm{Zn}} D \hbar \Omega_{\mathrm{Zn}} \gamma_{\mathrm{Zn}}}{B V_{0}}\right) \\
& -\frac{1}{2}\left(\frac{p_{\mathrm{O}} A_{\mathrm{O}}}{\Omega_{\mathrm{O}} M_{\mathrm{O}}}-\frac{p_{\mathrm{O}} D \hbar \Omega_{\mathrm{O}} \gamma_{\mathrm{O}}}{B V_{0}}\right) .
\end{aligned}
$$

Since $\Omega_{\mathrm{Zn}}$ and $\Omega_{\mathrm{O}}$ are inversely proportional to the square root of the corresponding isotopic masses, the two renormalization terms scale as $M_{\mathrm{Zn}}^{-1 / 2}$ and $M_{\mathrm{O}}^{-1 / 2}$, respectively. In contrast, at very high temperatures, when $k_{B} T \gg \hbar \Omega_{\mathrm{Zn}}, \hbar \Omega_{\mathrm{O}}$, the expression for the band gap becomes

$$
\begin{aligned}
E_{G}(T, M, P)= & E_{G}^{\infty}-\frac{p_{\mathrm{Zn}} k_{B} T}{\hbar}\left(\frac{A_{\mathrm{Zn}}}{\Omega_{\mathrm{Zn}}^{2} M_{\mathrm{Zn}}}-\frac{D \hbar \gamma_{\mathrm{Zn}}}{B V_{0}}\right) \\
& -\frac{p_{\mathrm{O}} k_{B} T}{\hbar}\left(\frac{A_{\mathrm{O}}}{\Omega_{\mathrm{O}}^{2} M_{\mathrm{O}}}-\frac{D \hbar \gamma_{\mathrm{O}}}{B V_{0}}\right),
\end{aligned}
$$

i.e., independent of isotopic mass.

\section{EXPERIMENTAL RESULTS AND DISCUSSION}

In our recent low-temperature experiments ${ }^{20}$ on singlecrystal $\mathrm{ZnO}$, we showed that modulated reflectivity, employing wavelength-, electro-, or photomodulation, yields three sharp spectroscopic features related to the excitonic band gaps at the $\Gamma$ point, corresponding to electronic transitions from the top three valence bands to the lowest conduction band. Using linearly polarized light, two low-energy transitions, labeled $A$ and $B$, are observed for polarization perpendicular to the $c$ axis of $\mathrm{ZnO}$ with the wurtzite structure and the high-energy transition, labeled $C$, for parallel polarization. ${ }^{20}$ These results are in agreement with the polarization selection rules predicted by group theory. ${ }^{28}$

In the present investigation, modulated reflectivity spectra were recorded using unpolarized light for a $\mathrm{ZnO}$ sample with natural isotopic composition in the temperature range from 10 to $400 \mathrm{~K}$. Figure 1 shows such spectra obtained with photomodulation. As the temperature increases, three sharp features, spectroscopically resolved at low temperatures, shift to lower energy and become broader. The closely spaced $A$ and $B$ features, corresponding to a spin-orbit splitting, merge together at $\sim 300 \mathrm{~K}$ and all three features become indistinguishable around $400 \mathrm{~K}$.

We have previously shown ${ }^{20}$ that the line shape of the wavelength-modulated reflectivity spectra of $\mathrm{ZnO}$ observed at liquid-He temperatures can be accounted for by a polariton model. ${ }^{29}$ The same polariton model is used in the present study to fit the photomodulated reflectivity spectra. In calculating the modulated reflectivity spectra, a simplifying assumption is made that the mechanically chopped, co-incident laser radiation modulates only the band gap, but does not affect all the other parameters used in the polariton model. ${ }^{20}$

The transverse excitonic energies determined for the $A, B$, and $C$ band gaps of $\mathrm{ZnO}$ by fitting the modulated reflectivity spectra to the polariton model are plotted as a function of temperature in Fig. 2. The experimental data, shown as sym- 


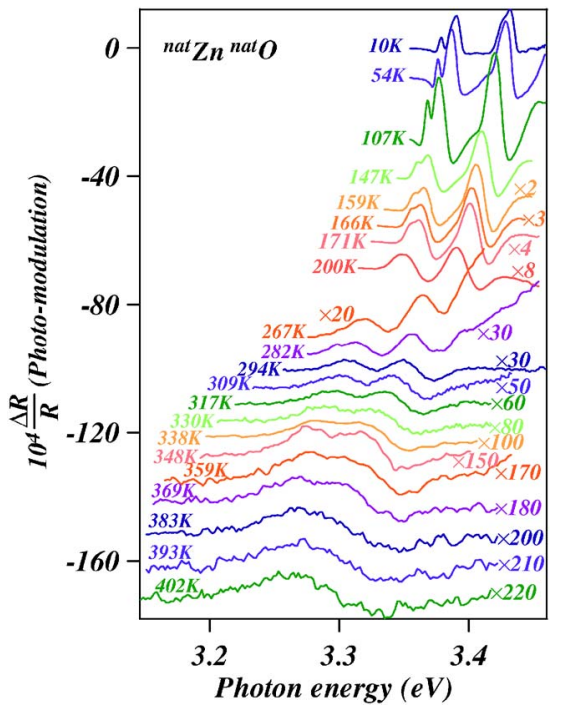

FIG. 1. (Color online) Photomodulated reflectivity spectra of ${ }^{n a t} \mathrm{Zn}^{\text {nat }} \mathrm{O}$ recorded in unpolarized light at different temperatures. For the convenience of the presentation the spectra were shifted vertically and multiplied by the factors shown in the figure.

bols, were fitted with a simplified version of Eq. (10), i.e.,

$$
\begin{aligned}
E_{G}(T, M, P)= & E_{G}^{\infty}+\frac{C_{\mathrm{Zn}}}{M_{\mathrm{Zn}}^{1 / 2}}\left(\frac{2}{\exp \left(\hbar \Omega_{\mathrm{Zn}} / k_{B} T\right)-1}+1\right) \\
& +\frac{C_{\mathrm{O}}}{M_{\mathrm{O}}^{1 / 2}}\left(\frac{2}{\exp \left(\hbar \Omega_{\mathrm{O}} / k_{B} T\right)-1}+1\right),
\end{aligned}
$$

where the coefficients $C_{\mathrm{Zn}}$ and $C_{\mathrm{O}}$ are given by

$$
\begin{aligned}
C_{\mathrm{Zn}} & =\left(-\frac{p_{\mathrm{Zn}} A_{\mathrm{Zn}}}{2 \Omega_{\mathrm{Zn}} M_{\mathrm{Zn}}^{1 / 2}}+\frac{p_{\mathrm{Zn}} D \hbar \Omega_{\mathrm{Zn}} \gamma_{\mathrm{Zn}} M_{\mathrm{Zn}}^{1 / 2}}{2 B V_{0}}\right), \\
C_{\mathrm{O}} & =\left(-\frac{p_{\mathrm{O}} A_{\mathrm{O}}}{2 \Omega_{\mathrm{O}} M_{\mathrm{O}}^{1 / 2}}+\frac{p_{\mathrm{O}} D \hbar \Omega_{\mathrm{O}} \gamma_{\mathrm{O}} M_{\mathrm{O}}^{1 / 2}}{2 B V_{0}}\right) .
\end{aligned}
$$

The notation in Eq. (14) is chosen to conform to that in Eq. (9) of Ref. 20.

For the fitting procedure, the average frequencies of the $\mathrm{Zn}$-dominated and O-dominated phonon modes were obtained as the weighted average frequencies of the acoustic and optic phonons, respectively, calculated for the zinc-

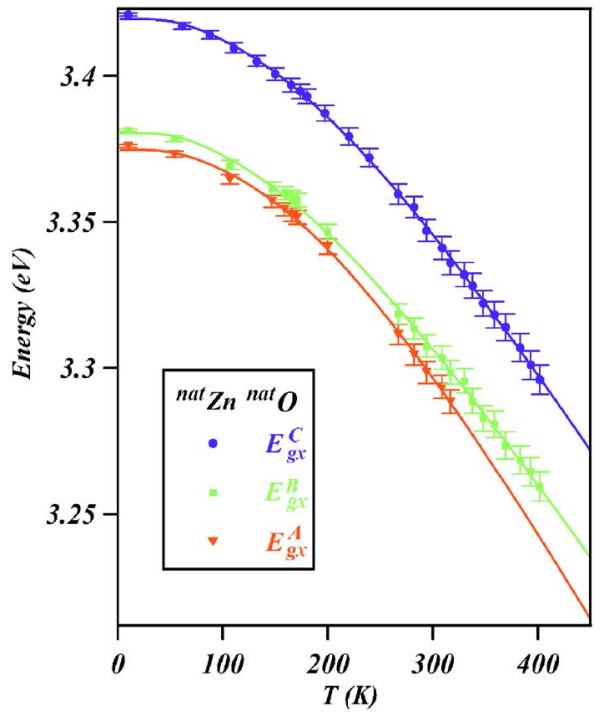

FIG. 2. (Color online) Transverse excitonic energies for the $A$, $B$, and $C$ band gaps of ${ }^{n a t} \mathrm{Zn}^{\text {nat }} \mathrm{O}$ plotted as functions of temperature. The symbols represent experimental data, whereas the solid lines are two-oscillator fits to Eq. (14).

blende modification of $\mathrm{ZnO}$ and shown in Table VII of Ref. $30\left(\hbar \Omega_{\mathrm{Zn}}=20.1 \mathrm{meV}\right.$ and $\left.\hbar \Omega_{\mathrm{O}}=63.2 \mathrm{meV}\right)$. The band gap for the static lattice $E_{G}^{\infty}$ and the coefficients $C_{\mathrm{Zn}}$ and $C_{\mathrm{O}}$, in turn, were treated as fitting parameters. The parameters determined from the fits for the $A, B$, and $C$ excitonic band gaps are summarized in Table I. In addition, the fifth column of the table shows zero-point renormalization of the band gaps given through Eq. (14) by $-\left(C_{\mathrm{Zn}} / M_{\mathrm{Zn}}^{1 / 2}+C_{\mathrm{O}} / M_{\mathrm{O}}^{1 / 2}\right)$, whereas the last column contains the slope of the linear portion of the temperature dependence of the band gap expected at high temperatures, i.e., when $k_{B} T \gg \hbar \Omega_{\mathrm{Zn}, \mathrm{O}}$. The latter quantity is calculated as $\left(2 k_{B} C_{\mathrm{Zn}} / \hbar \Omega_{\mathrm{Zn}} M_{\mathrm{Zn}}^{1 / 2}\right.$ $+2 k_{B} C_{\mathrm{O}} / \hbar \Omega_{\mathrm{O}} M_{\mathrm{O}}^{1 / 2}$ ).

The $B$ excitonic band gap of the static lattice and the zero-point renormalizations of the $B$ and $C$ gaps, deduced in this manner for the ${ }^{n a t} \mathrm{Zn}^{\text {nat }} \mathrm{O}$, are in excellent agreement with those obtained from the low-temperature isotopic study ${ }^{20}$ of $\mathrm{ZnO}$. In additon, very close values were determined in the present and isotopic studies for the $C$ excitonic band gap of the infinitely massive isotope. The $A$ excitonic band gap of the static lattice and its renormalization obtained in the

TABLE I. Parameters determined from fits of the temperature dependence of the band gap to Eq. (14).

\begin{tabular}{lccccc}
\hline \hline $\begin{array}{l}\text { Band gap } \\
\text { (sample) }\end{array}$ & $\begin{array}{c}E_{G}^{\infty} \\
(\mathrm{eV})\end{array}$ & $\begin{array}{c}C_{\mathrm{Zn}} \\
\left(\mathrm{meV} \mathrm{amu}{ }^{1 / 2}\right)\end{array}$ & $\begin{array}{c}C_{\mathrm{O}} \\
\left(\mathrm{meV} \mathrm{amu}{ }^{1 / 2}\right)\end{array}$ & $\begin{array}{c}E_{G}^{\infty}-E_{G}(T=0) \\
(\mathrm{meV})\end{array}$ & $\begin{array}{c}\left(d E_{G} / d T\right)_{k_{B} T \hbar \Omega} \\
(\mathrm{meV} / \mathrm{K})\end{array}$ \\
\hline \multicolumn{5}{c}{ Photomodulated reflectivity } \\
$A\left({ }^{n a t} \mathrm{Zn}^{n a t} \mathrm{O}\right)$ & $3.555 \pm 0.005$ & $-235 \pm 16$ & $-608 \pm 16$ & $181 \pm 6$ & $-0.664 \pm 0.028$ \\
$B\left({ }^{n a t} \mathrm{Zn}^{n a t} \mathrm{O}\right)$ & $3.517 \pm 0.005$ & $-259 \pm 16$ & $-420 \pm 20$ & $137 \pm 7$ & $-0.561 \pm 0.031$ \\
$C\left({ }^{n a t} \mathrm{Zn}^{n a t} \mathrm{O}\right)$ & $3.572 \pm 0.005$ & $-235 \pm 24$ & $-492 \pm 20$ & $152 \pm 8$ & $-0.584 \pm 0.039$ \\
$C\left({ }^{n a t} \mathrm{Zn}^{n a t} \mathrm{O}\right)$ & $3.569 \pm 0.004$ & $-235 \pm 24$ & $-480 \pm 16$ & $149 \pm 7$ & $-0.576 \pm 0.037$ \\
$C\left({ }^{6} \mathrm{Zn}^{18} \mathrm{O}\right)$ & $3.568 \pm 0.003$ & $-264 \pm 25$ & $-458 \pm 17$ & $140 \pm 7$ & $-0.569 \pm 0.038$ \\
\hline \hline
\end{tabular}


TABLE II. Relative contributions of the electron-phonon interaction and the thermal expansion to the coefficients $C_{\mathrm{Zn}}$ and $C_{\mathrm{O}}$.

\begin{tabular}{|c|c|c|c|c|}
\hline $\begin{array}{l}\text { Band gap } \\
\text { (sample) }\end{array}$ & $\frac{p_{\mathrm{Zn}} D \hbar \Omega_{\mathrm{Zn}} \gamma_{\mathrm{Zn}} M_{\mathrm{Zn}}^{1 / 2}}{2 B V_{0}}$ & $-\frac{p_{\mathrm{Zn}} A_{\mathrm{Zn}}}{2 \Omega_{\mathrm{Zn}} M_{\mathrm{Zn}}^{1 / 2}}$ & $\frac{p_{\mathrm{O}} D \hbar \Omega_{\mathrm{O}} \gamma_{\mathrm{O}} M_{\mathrm{O}}^{1 / 2}}{2 B V_{0}}$ & $\begin{array}{c}-\frac{p_{\mathrm{O}} A_{\mathrm{O}}}{2 \Omega_{\mathrm{O}} M_{\mathrm{O}}^{1 / 2}} \\
\left(\mathrm{meV} \mathrm{amu}^{1 / 2}\right)\end{array}$ \\
\hline$A\left({ }^{n a t} \mathrm{Zn}^{n a t} \mathrm{O}\right)$ & 34 & $-269 \pm 16$ & -93 & $-515 \pm 16$ \\
\hline$B\left({ }^{n a t} \mathrm{Zn}^{n a t} \mathrm{O}\right)$ & 35 & $-294 \pm 16$ & -96 & $-314 \pm 20$ \\
\hline$C\left({ }^{n a t} \mathrm{Zn}^{n a t} \mathrm{O}\right)$ & 38 & $-273 \pm 24$ & -104 & $-388 \pm 20$ \\
\hline
\end{tabular}

present work are different from those of the isotopic investigation. This probably results from the limited range of the temperature data available for the $A$ band gap, as a consequence of the spectroscopic feature $A$ being completely blocked by the close by, stronger feature $B$ for temperatures higher than $300 \mathrm{~K}$ (see Fig. 1). To summarize, the analysis of the temperature and isotopic dependence of the $A, B$, and $C$ excitonic band gaps of $\mathrm{ZnO}$ in terms of the two-oscillator model yields consistent results.

Using parameters appropriate for zinc-blende $\mathrm{ZnO}$, relative contributions of the electron-phonon interaction and the thermal expansion to the temperature dependence of the band gap can be estimated. The thermal-expansion contribution is given by the term $\left(p D \hbar \Omega \gamma M^{1 / 2}\right) /\left(2 B V_{0}\right)$ in Eqs. (15), or $-\left(p \hbar \Omega \gamma M^{1 / 2} / 2 V_{0}\right)\left(\partial E_{G} / \partial P\right)_{T, M}$ when the relationship $D=-B\left(\partial E_{G} / \partial P\right)_{T, M}$ is utilized. With $\gamma_{\mathrm{Zn}} \approx-0.9$, $\gamma_{\mathrm{O}} \approx 1.6, V_{0}=24.65 \AA^{3}$ (from Tables I and VII of Ref. $30), p_{\mathrm{Zn}}=p_{\mathrm{O}}=3$, and $\left(\partial E_{g x}^{A} / \partial P\right)_{T, M}=23.6 \mathrm{meV} / \mathrm{GPa},\left(\partial E_{g x}^{B} /\right.$ $\partial P)_{T, M}=24.4 \mathrm{meV} / \mathrm{GPa}, \quad\left(\partial E_{g x}^{C} / \partial P\right)_{T, M}=26.5 \mathrm{meV} / \mathrm{GPa},{ }^{31}$ the thermal-expansion contributions to the temperature dependence of the $A, B$, and $C$ band gaps of ${ }^{n a t} \mathrm{Zn}^{\text {nat }} \mathrm{O}$ are calculated and shown in the second and fourth columns of Table II. The electron-phonon contributions given in columns III and V are obtained by subtracting the corresponding thermal-expansion contributions from the coefficients $C_{\mathrm{Zn}}$ and $C_{\mathrm{O}}$.

The results presented in Table II are in good agreement with those obtained in the isotopic study (Table IV of Ref. 20). For instance, the electron-phonon and the thermalexpansion contributions to the Zn-related term in Eq. (14) have opposite signs, with the magnitude of the former being approximately an order of magnitude larger than that of the latter. The O-related term is dominated by the electronphonon contribution, similar to the results of the isotopic study, ${ }^{20}$ although the relative strengths of the two contributions are slightly different in the present and the isotopic investigations. The positive thermal-expansion contribution to the $\mathrm{Zn}$-related term is a result of the negative average Grüneisen parameter for acoustic phonons. ${ }^{30}$

As was already mentioned in Refs. 17 and 20, the contribution of the oxygen vibrations to the gap renormalization at $T=0$ is about five times larger than that of the zinc vibrations. Such large renormalization is characteristic of materials with atoms from the second row of the periodic table $(\mathrm{C}$, $\mathrm{N}, \mathrm{O}){ }^{5,32,33}$ This fact reflects a large electron-phonon interaction at the top valence band of semiconductors containing such elements (e.g., diamond, $\mathrm{GaN}, \mathrm{ZnO}$ ) which has been shown to lead to superconductivity in boron-doped diamond. ${ }^{34}$ The corresponding effect in silicon has been recently observed to exhibit a much lower $T_{c}\left(T_{c} \sim 0.3 \mathrm{~K}\right)$ as it corresponds to a lower phonon-hole interaction. ${ }^{35}$ Because of the phonon-hole interaction related to the presence of oxygen, $p$-type $\mathrm{ZnO}$ may show superconductivity at a higher $T_{c}$ or a lower hole concentration than reported for $\mathrm{Si}^{32}$

In a separate experimental effort, we made an attempt to verify the decreasing isotopic dependence of the band gap with increasing temperature predicted in Eqs. (12) and (13). The small size of the isotopically engineered sample necessitated the use of the wavelength-modulation technique. Figure 3 compares the wavelength-modulated reflectivity spectra recorded at the same temperatures for ${ }^{n a t} \mathrm{Zn}^{n a t} \mathrm{O}$ and ${ }^{68} \mathrm{Zn}^{18} \mathrm{O}$ with light polarized along the $c$ axis. The observed spectral features are associated with the $C$ excitonic band gap, the vertical lines in the figure indicating the positions of the transverse $C$ excitons as determined by fitting the spectra to the polariton model. ${ }^{20}$ The wavelength-modulated reflectivity spectra in Fig. 3 clearly demonstrate that the difference

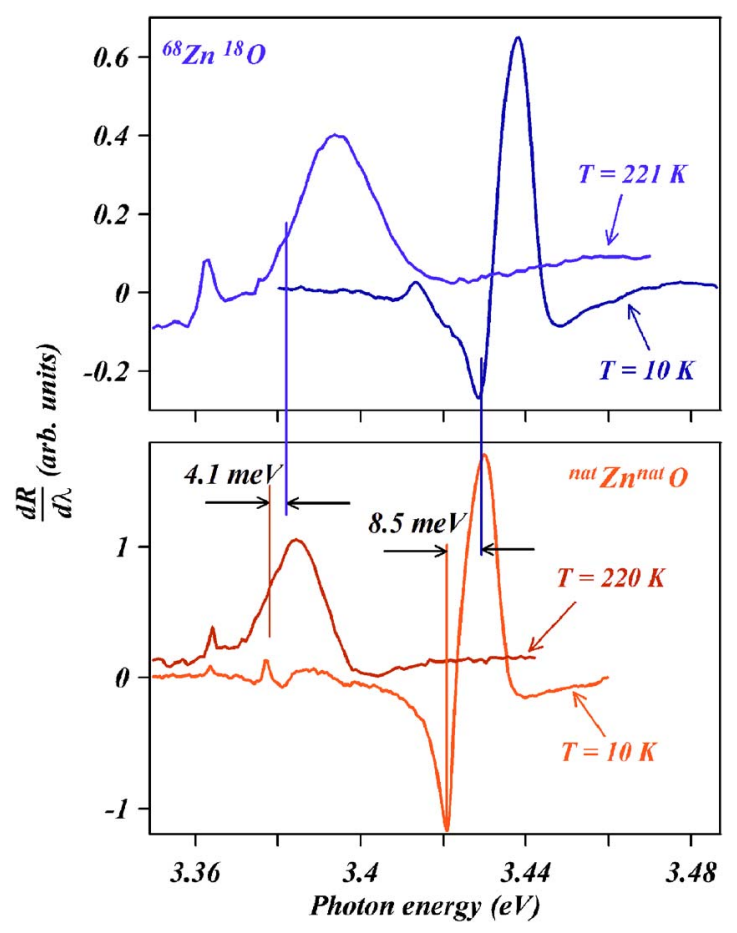

FIG. 3. (Color online) Wavelength-modulated reflectivity spectra of ${ }^{\text {nat }} \mathrm{Zn}^{\text {nat }} \mathrm{O}$ and ${ }^{68} \mathrm{Zn}^{18} \mathrm{O}$. The vertical lines indicate positions of the transverse $C$ excitonic band gap determined from the line-shape analysis based on the polariton model. 


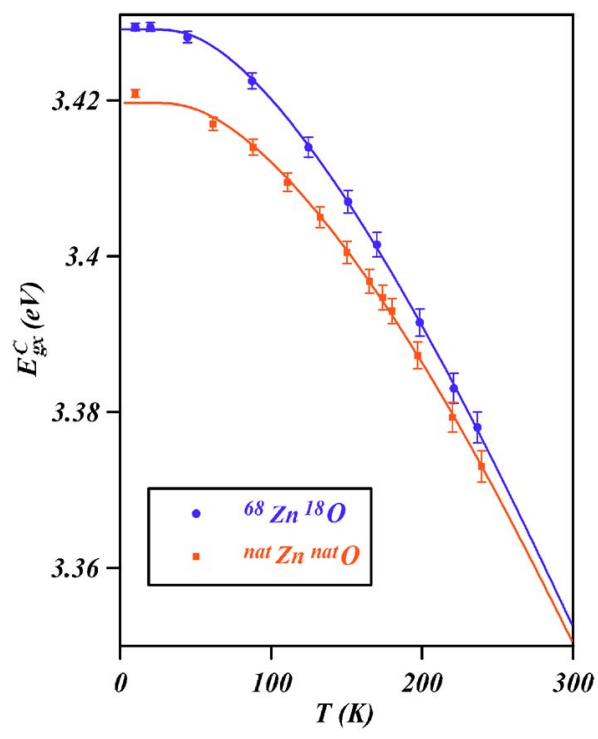

FIG. 4. (Color online) The transverse $C$ excitonic band gap as a function of temperature in ${ }^{n a t} \mathrm{Zn}^{\text {nat }} \mathrm{O}$ and ${ }^{68} \mathrm{Zn}^{18} \mathrm{O}$. Symbols are experimental data obtained from the fits of WMR spectra to the polariton model and lines are two-oscillator fits to Eq. (14).

in the $C$ band gap in the two samples decreases with increasing temperature.

The transverse $C$ excitonic energy deduced from the wavelength-modulated reflectivity spectra of ${ }^{n a t} \mathrm{Zn}^{\text {nat }} \mathrm{O}$ and ${ }^{68} \mathrm{Zn}^{18} \mathrm{O}$ in the temperature range $10-240 \mathrm{~K}$ is plotted in Fig. 4 as a function of temperature. Experimental data, shown by symbols, were fitted with Eq. (14), the corresponding solid lines representing the fits, the fitting parameters being included in Table I. For the purpose of the fit, the average frequencies of $\mathrm{Zn}$ - and $\mathrm{O}$-dominated phonon modes in ${ }^{68} \mathrm{Zn}^{18} \mathrm{O}$ were obtained as those in ${ }^{\text {nat }} \mathrm{Zn}^{\text {nat }} \mathrm{O}$ (see above) multiplied by $\left[M\left({ }^{n a t} \mathrm{Zn}\right) / M\left({ }^{68} \mathrm{Zn}\right)\right]^{1 / 2}$ and $\left[M\left({ }^{n a t} \mathrm{O}\right) / M\left({ }^{18} \mathrm{O}\right)\right]^{1 / 2}$, respectively. The solid lines explicitly demonstrate convergence of the $C$ band gap in the two $\mathrm{ZnO}$ samples with increasing temperature, thus confirming the theoretically predicted trend for the isotopic dependence of the band gap on temperature. ${ }^{36}$ In addition, the slopes of the temperature dependence of the $C$ excitonic band gap in ${ }^{n a t} \mathrm{Zn}^{\text {nat }} \mathrm{O}$ and ${ }^{68} \mathrm{Zn}^{18} \mathrm{O}$ calculated at high temperatures using the fitting parameters (Table I) are in excellent agreement, supporting the independence of the band gap from the isotopic mass at high temperatures.

\section{CONCLUSIONS}

We have investigated the temperature dependence of the $A, B$, and $C$ excitons of $\mathrm{ZnO}$ from low to high temperatures. The experimental temperature data led to zero-point renormalization of the band gaps of $\mathrm{ZnO}$ with natural isotopic composition which agree with those determined through isotopic substitution in Refs. 17 and 20. These results verify the equivalence of both methods of obtaining zero-point renormalizations of energy gaps.

The temperature dependence of the band gap is compared for two samples with different isotopic compositions. The difference in their $C$ gap renormalizations decreases with increasing temperature and approaches zero for $T$ tending to infinity, as expected for the classical limit of the electronphonon interaction. A fit to the measured temperature dependence of the $A, B$, and $C$ excitons, using a two oscillator model,${ }^{37}$ reveals that the interaction of the gap electrons with the oxygenlike component of the lattice vibrations is much stronger than that with the zinclike component, thus reproducing the behavior observed for other semiconductors containing elements of the second row of the periodic table (GaN, diamond). Recent observations of superconductivity in $p$-type diamond $\left(T_{c} \sim 10 \mathrm{~K}\right)$ and silicon $\left(T_{c} \sim 0.3 \mathrm{~K}\right)$ can be explained on the basis of this hypothesis. They suggest the possible appearance of superconductivity in $\mathrm{ZnO}$ at temperatures between 0.3 and $10 \mathrm{~K}$, provided one succeeds in doping the material $p$-type with hole concentrations around $5 \times 10^{20}$ holes $/ \mathrm{cm}^{3}$.

\section{ACKNOWLEDGMENT}

The authors at Purdue thank the National Science Foundation for support through Grants No. DMR-0102699 and DMR-0405082.
*Present address: Center for Bio/Molecular Science and Engineering, Naval Research Laboratory, Washington, DC 20375.

${ }^{1}$ M. Becker and H. Y. Fan, Phys. Rev. 76, 1531 (1949); H. Y. Fan and M. Becker, Phys. Rev. B 78, 178 (1950).

${ }^{2}$ H. Y. Fan, Phys. Rev. 78, 808 (1950).

${ }^{3}$ H. Y. Fan, Phys. Rev. 82, 900 (1951).

${ }^{4}$ E. Antončik, Czech. J. Phys. 5, 449 (1955).

${ }^{5}$ See, for example, M. Cardona, Solid State Commun. 133, 3 (2005).

${ }^{6}$ M. L. Cohen and J. R. Chelikowski, Electronic Structure and Optical Properties of Semiconductors (Springer-Verlag, Heidelberg, 1988).

${ }^{7}$ A. T. Collins, S. C. Lawson, G. Davies, and H. Kanda, Phys. Rev. Lett. 65, 891 (1990).
${ }^{8}$ G. Davies, E. C. Lightowlers, K. Itoh, W. L. Hansen, E. E. Haller, and V. Ozhogin, Semicond. Sci. Technol. 7, 1271 (1992).

${ }^{9}$ C. Parks, A. K. Ramdas, S. Rodriguez, K. M. Itoh, and E. E. Haller, Phys. Rev. B 49, 14244 (1994).

${ }^{10}$ P. Etchegoin, J. Weber, M. Cardona, W. L. Hansen, K. Itoh, and E. E. Haller, Solid State Commun. 83, 843 (1992).

${ }^{11}$ D. Karaiskaj, M. L. W. Thewalt, T. Ruf, M. Cardona, and M. Konuma, Solid State Commun. 123, 87 (2002).

${ }^{12}$ S. Tsoi, H. Alawadhi, X. Lu, J. W. Ager III, C. Y. Liao, H. Riemann, E. E. Haller, S. Rodriguez, and A. K. Ramdas, Phys. Rev. B 70, 193201 (2004).

${ }^{13}$ See T. A. Meyer, M. L. W. Thewalt, M. Cardona, and R. Lauck, Phys. Rev. B 69, 115214 (2004), and references therein.

${ }^{14}$ See S. R. Ahuja, L. Farst, O. Ericson, J. M. Willis, and B. Jo- 
hannson, J. Appl. Phys. 83, 8065 (1998), and references therein.

${ }^{15}$ C. Boemare, T. Monteiro, M. J. Soares, J. G. Guilherme, and E. Alves, Physica B 308-310, 985 (2001).

${ }^{16}$ Y. Harada, H. Kondo, N. Ichimura, and S. Hashimoto, Jpn. J. Appl. Phys., Part 2 38, L1318 (1999).

${ }^{17}$ F. J. Manjón, M. Mollar, M. A. Hernández-Fenollosa, B. Marí, R. Lauck, and M. Cardona, Solid State Commun. 128, 35 (2003).

${ }^{18}$ L. Wang and N. C. Giles, J. Appl. Phys. 94, 973 (2003).

${ }^{19}$ F. I. Kreingol'd and B. S. Kulinkin, Sov. Phys. Solid State 28, 1781 (1986); Fiz. Tverd. Tela (Leningrad) 28, 3164 (1986).

${ }^{20}$ S. Tsoi, X. Lu, A. K. Ramdas, H. Alawadhi, M. Grimsditch, M. Cardona, and R. Lauck, Phys. Rev. B 74, 165203 (2006).

${ }^{21}$ M. Y. Hu, H. Sinn, A. Alatas, W. Sturhahn, E. E. Alp, H.-C. Willie, Yu. V. Shvyd'ko, J. P. Sutter, J. Bandaru, E. E. Haller, V. I. Ozhogin, S. Rodriguez, R. Colella, E. Kartheuser, and M. A. Villeret, Phys. Rev. B 69, 079902(E) (2004).

${ }^{22}$ A. Göbel, T. Ruf, M. Cardona, C. T. Lin, J. Wrzesinski, M. Steube, K. Reimann, J.-C. Merle, and M. Joucla, Phys. Rev. B 57, 15183 (1998).

${ }^{23}$ A. Debernardi and M. Cardona, Phys. Rev. B 54, 11305 (1996).

${ }^{24}$ J. M. Zhang, T. Ruf, R. Lauck, and M. Cardona, Phys. Rev. B 57, 9716 (1998).

${ }^{25}$ R. Pässler, J. Appl. Phys. 89, 6235 (2001).

${ }^{26}$ In the zinc-blende structure, these are the acoustic modes; in wurtzite, with two molecular units per primitive cell, these modes include acoustic and folded acoustic components, i.e., the three lowest optical-phonon branches.
${ }^{27}$ Except at very low temperatures where the renormalization has an additional factor of $\Omega^{2}$ and thus becomes proportional to $T^{4}$. See M. Cardona, T. A. Meyer, and M. L. W. Thewalt, Phys. Rev. Lett. 92, 196403 (2004).

${ }^{28}$ J. L. Birman, Phys. Rev. 114, 1490 (1959).

${ }^{29}$ J. J. Hopfield and D. G. Thomas, Phys. Rev. 132, 563 (1963).

${ }^{30}$ J. Serrano, A. H. Romero, F. J. Manjón, R. Lauck, M. Cardona, and A. Rubio, Phys. Rev. B 69, 094306 (2004).

${ }^{31}$ A. Mang, K. Reimann, and St. Rübenacke, Solid State Commun. 94, 251 (1995).

${ }^{32}$ M. Cardona, Sci. Technol. Adv. Mater. 7S1, 60 (2006).

${ }^{33}$ M. Cardona and M. L. W. Thewalt, Rev. Mod. Phys. 77, 1173 (2005).

${ }^{34}$ E. A. Ekimov, V. A. Sidorov, E. D. Bauer, N. N. Mel'nik, N. J. Curro, J. D. Thompson, and S. M. Stishov, Nature (London) 428, 542 (2004).

${ }^{35}$ E. Bustarret, C. Marcenat, P. Achatz, J. Kačmarčik, F. Lévy, A. Huxley, L. Ortéga, E. Bourgeois, X. Blase, D. Débarre, and J. Boulmer, Nature (London) 444, 465 (2006).

${ }^{36}$ A. Einstein and O. Stern, Ann. Phys. (Leipzig) 40, 551 (1913). In this article the authors suggest that the zero-point energy of a harmonic oscillator equals $\hbar \Omega / 2$, and thus is proportional to $M^{-1 / 2}, M$ being the mass of the oscillator. They also show that at high $T$ the oscillator energy tends to $k_{B} T$, i.e., becomes independent of the mass.

${ }^{37}$ W. Nernst and F. A. Lindemann, Z. Elektrochem. Angew. Phys. Chem. 18, 817 (1911). 